Supporting Information for:

\title{
High-Pressure, High-Temperature Synthesis and Characterization of Polar and Magnetic $\mathrm{LuCrWO}_{6}$
}

Sun Woo Kim ${ }^{1,2}$, Xiaoyan Tan ${ }^{2, \#}$ Corey E. Frank ${ }^{2}$, Zheng Deng ${ }^{3}$, Huaiyu Wang ${ }^{4}$, Liam Collins ${ }^{5}$, Saul H. Lapidus ${ }^{6}$, Changqing Jin ${ }^{3}$, Venkatraman Gopalan ${ }^{4}$, Sergei V. Kalinin ${ }^{5}$, David Walker ${ }^{7}$, and Martha Greenblatt ${ }^{2, *}$

1. Department of Chemistry Education, Chosun University, Gwangju 61452, South Korea

2. Department of Chemistry and Chemical Biology, Rutgers, the State University of New Jersey, 610 Taylor Road, Piscataway, NJ 08854 USA

3. Beijing National Laboratory for Condensed Matter Physics, Institute of Physics, Chinese Academy of Sciences, Beijing 100190, China

4. Department of Materials Science and Engineering, Pennsylvania State University, University Park, Pennsylvania 16802, USA

5. Center for Nanophase Material Science \& Institute for Functional Imaging Materials, Oak Ridge National Laboratory, Oak Ridge, Tennessee 37831, USA

6. Advanced Photon Source, Argonne National Laboratory, Lemont, Illinois 60439, USA

7. Lamont-Doherty Earth Observatory, Columbia University, Palisades, NY 10964, USA

\#Current address: Department of Chemistry and Biochemistry, George Mason University, Fairfax, Virginia 22030, USA

Email: greenbla@chem.rutgers.edu 
(a)

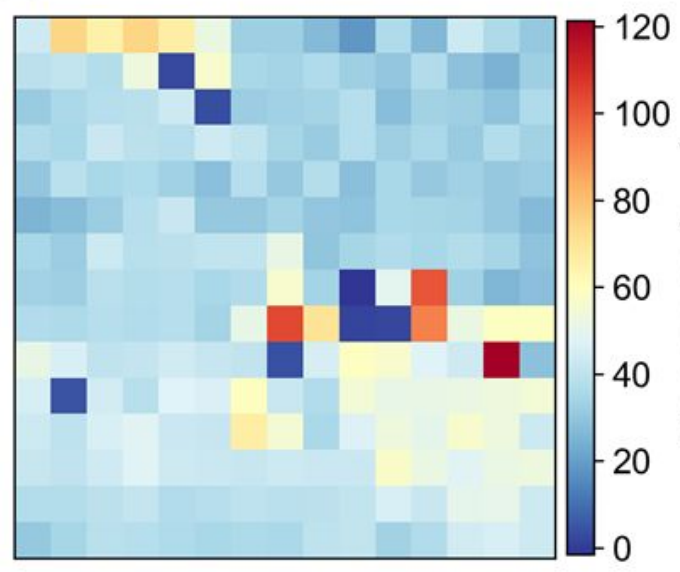

(b)

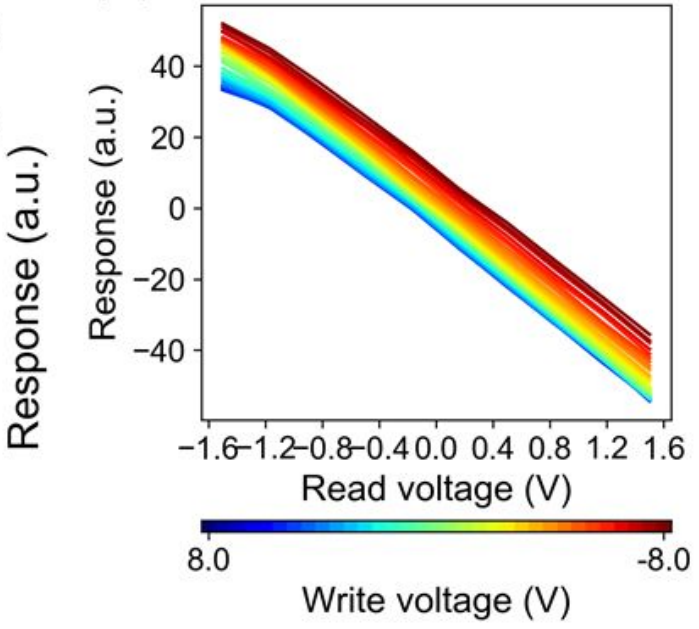

Figure S1. Contact resonance KPFM (a) 15 x 15 grid location and (b) average cKPFM curve. 


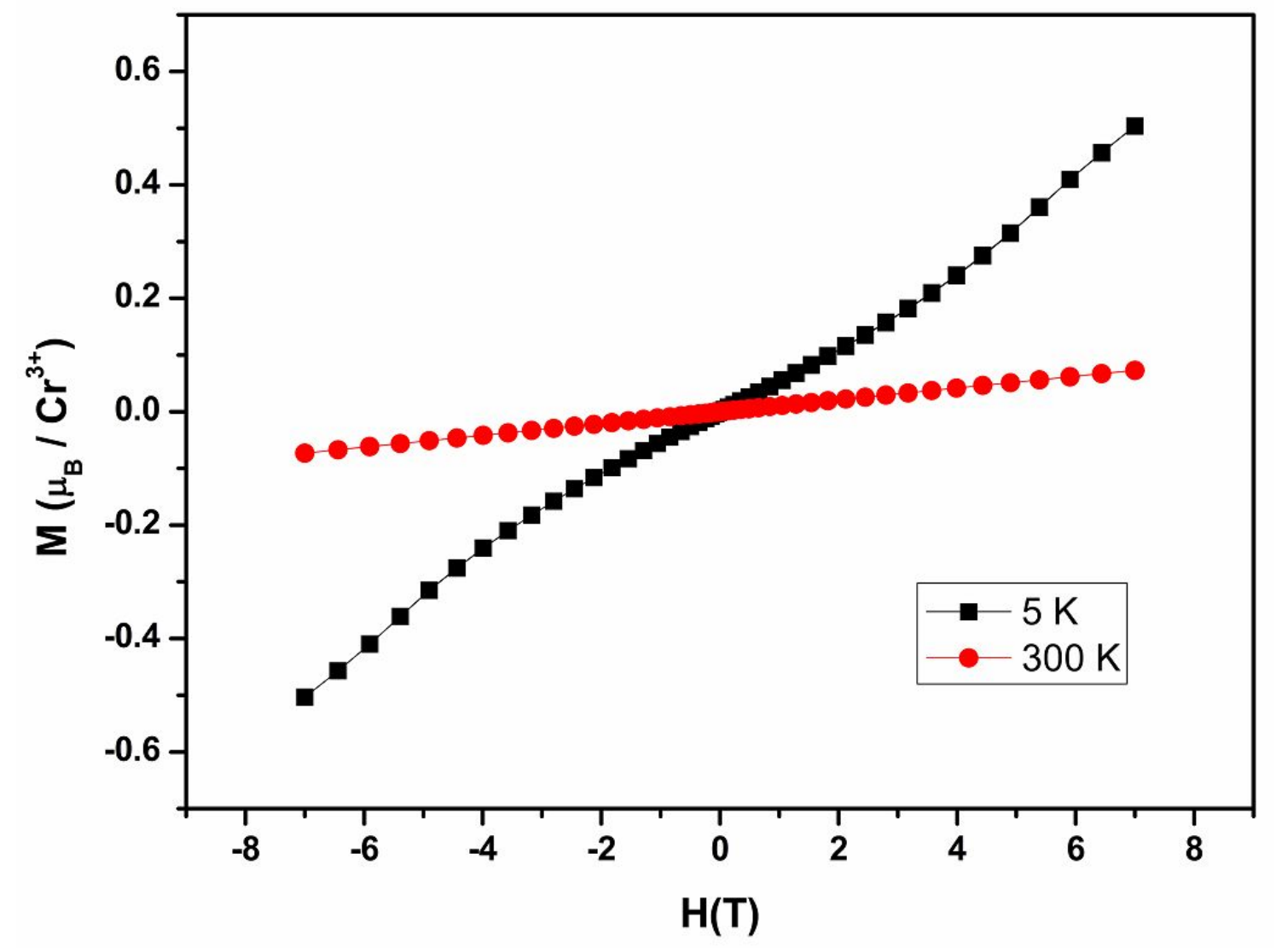

Figure S2. Isothermal magnetization of $\mathrm{LuCrWO}_{6}$ measured at 5 and $300 \mathrm{~K}$ as a function of applied field $H$. 